\title{
Production of human polyclonal antibodies by transgenic animals
}

\author{
Louis-Marie Houdebine
}

Department of Biology of Development and Reproduction, National Institute of Agronomical Research, Jouy en Josas, France. E-mail: louis.houdebine@jouy.inra.fr

Received 9 February 2011; revised 5 April 2011; accepted 6 April 2011.

\begin{abstract}
Polyclonal antibodies collected from the blood of animals and humans experimentally immunised or spontaneously immunised respectively can be injected into patients to protect them against pathogens, toxins, tumours etc. This approach is severely limited by the availability of human polyclonal antibodies of interest. Moreover, polyclonal antibodies from animals are recognised as antigens by patients and are thus rapidly rejected and inactivated. To circumvent this problem, animals (essentially rabbits, chicken, pigs and cows) are being genetically engineered. Their immunoglobulin genes are being inactivated and the corresponding human immunoglobulin genes are being transferred to them. These animals will be immunized and it is expected that large amounts of pure human polyclonal antibodies will be extracted from their blood to be administered to patients. The possible acceptability problem of this approach is under a case study of the European Union Pegasus project.
\end{abstract}

Keywords: Human Polyclonal Antibodies; Transgenic Animals; Acceptability

\section{INTRODUCTION}

A natural immunisation or a vaccination implies the generation of multiple antibodies (Abs) recognising each a specific region of the antigen, known as epitope. Each $\mathrm{AB}$ is known as a monoclonal antibody (MAB) as it is synthesised by a single clone of $\mathrm{B}$ lymphocytes. The multiple ABs recognising the different epitopes of the same antigen are circulating in blood and they are known as polyclonal antibodies (PAB). PABs are thus the sum of all the monoclonal antibodies generated during an immunisation. Each MAB activates defence mechanisms and PABs generated after spontaneous and experimental immunisations are thus generally more potent than MABs. PABs can be classically obtained essentially from humans or from animals previously immunised by spontaneous infections or vaccinations. In practice, the use of PABs is limited by the low availability of human PABs but also by the fact that animal PABs are recognized as foreign antigens by patients who develop immune reactions against them.

Several decades ago, MABs targeting specific antigens appeared as potential "magic bullets" able to neutralise pathogens, to inhibit biological functions and to prevent tumour cell multiplication. The possibility to bind MAB to various molecules toxic for tumour cells in order to enhance their effects appeared also attractive. This implied the implementation of methods making it possible the preparation of MABs in sufficient amount. This method is based on the isolation of B lymphocyte clones secreting each a single MAB. The cellular clones (hybridoma) able to secrete one MAB may be cultured in fermentors or implanted into mice. The first method allows the preparation of sufficient quantity of MAB for experimentation but not for pharmaceutical treatments, as hybridoma are unstable. The second method which implies the development of mouse tumours secreting the MAB is no more used for ethical reasons. The alternative consists of isolating the MAB genes from the hybridoma and to transfer them into cells (generally $\mathrm{CHO}$, Chinese hamster ovary cells) that can be cultured in large amount in fermentors. Another possibility is to transfer the MAB genes into animals or plants and collect the recombinant MABs from milk, egg white, leaves or grains [1-3].

The major drawback of mouse MABs is that they are foreign molecules and thus antigens inducing in patients the formation of human antibodies against the mouse MABs which become inactivated. To circumvent this problem, the mouse MABs may be engineered to replace their constant domain by the human counterpart. The resulting MABs are known as chimeric MABs. The mouse antibody sequences are then limited to the variable regions and chimeric MAB can be used as thera- 
peutics without being rapidly rejected. In 2007, 18 chimeric MABs were commercialized.

Ideally, fully human MABs should be used. To reach this goal, mice have been engineered in such a way as to harbour the human immunoglobulin genes but no more their own immunoglobulin genes. The immunisation of these mice induces the formation of strictly human PABs. Human MABs can be prepared from these mice following the method depicted above [4].

\section{DIFFERENT STEPS OF THE GENETIC MODIFICATION}

The transgenic mice depicted above produce human PABs in their blood after having been immunized. $\mathrm{Hu}-$ man PABs directed against a specific antigen can thus be extracted from these mice. Yet, mice are too small animals to be the source of PABs for human therapy. Larger animals are thus being implemented to provide patients with human PABs extracted from the animal blood.

Mice in which their immunoglobulin genes have been inactivated and harbouring the human immunoglobulin genes are currently used to obtain fully human MABs. The proof of concept is thus strongly established. The preparation of human PABs from rabbits, chicken, pigs and cows is thus expected to face essentially technical and cost problems. The different steps of the project are the followings.

1) Inactivation of the animal immunoglobulin genes

This must be achieved for two reasons. The human genes integrated into the animal genome enter in competition with the corresponding animal genes leading to a reduced expression of the human genes. The second reason is that the simultaneous presence of the two sets of genes leads to the generation of fully human, fully animal and hybrid human-animal antibodies (for example antibodies containing a human heavy chain and an animal light chain). The hybrid antibodies are functional but not appropriate for human therapy. The inactivation of the animal immunoglobulin genes can be performed by the conventional homologous recombination method (known as knock out) in cells further used to generate the genetically engineered animals. These cells may be pluripotent ES cells able to transfer the genetic modification to progeny after having been introduced into early embryos. Alternatively, the gene knock out may be carried out in somatic cells able to transfer the genetic modification to progeny using SCNT (somatic cell nuclear transfer) [5]. Alternatively, the genes can be inactivated by a cleavage of DNA at chosen sites within the immunoglobulin genes followed by an imperfect DNA repair (NHEJ: non homologous end joining) leading to a targeted gene inactivation. The DNA cleavage is achieved by meganucleases, ZFN (Zinc Finger Nucleases) or
TALE (transcription activator-like effector) nucleases which are endonucleases engineered to recognize specific DNA sequences in a genome and to cleave both DNA strands at this site. This method proved to be efficient by injecting the ZFN or the TALE directly into embryos [6,7].

Some of the immunoglobulin genes have been inactivated by homologous recombination and cloning in cows [5]. The cows which will be used are those in which the prion gene PrP has been inactivated $[8,9]$. Preexisting natural lines of rabbits having inactive immunoglobulin genes are also being used. The inactivation of the other rabbit immunoglobulin genes will have to be achieved by NHEJ (experiment in course) or conventional knock out.

2) Addition of human antibody genes

The human immunoglobulin gene addition was achieved by the SCNT method in cows and by DNA microinjection in rabbit embryos. The human immunoglobulin genes which are contained in a fragment of the human genome were thus added randomly to the animal genome and they did not replace the animal genes strictly speaking.

3) Immunisation of the animals

Conventional immunisation methods will be used. Several lines of transgenic animals will be established and available to be immunized against any antigens. They will be kept in pathogen free areas according to existing regulations. Bleeding of the immunised animals will provide with large amounts of blood. This step does not imply the sacrifice of the animals which may be used for months or years to produce given PABs. In order to have a constant concentration and activity of PABs, numerous samples will be mixed before $\mathrm{PAB}$ purification.

4) Purification and validation of $P A B s$

PABs will be purified from blood by well-established methods. Their purity and their efficiency will be assessed following conventional methods and regulations [10].

5) Commercialisation of PABs

The commercialisation will be achieved essentially as PABs extracted from human or animal blood and MABs are currently commercialised. The generation of the transgenic animals represents a relatively heavy initial investment in time and money, especially in cows. These animals will be universal tools to obtain PABs against any antigen of interest. The production of human PABs is thus expected to be of a low cost. Their purification and validation will represent the major cost.

\section{GENERATION OF THE GM ANIMALS: DEGREE OF ACHIEVEMENT IN 2011}

Four species have been retained for this project: cows, pigs, rabbits and chicken [11-15]. Cows are appropriate 
to produce large amount of human PABs. Rabbits are already used in routine to prepare polyclonal antibodies to prevent grafted organ rejection and to obtain antithymoglobulin antibodies, both being used in humans. Rabbits may be bred at a low cost in pathogen free facilities. This approach is thus considered as safe. Moreover, transgenesis and breeding are simpler and cheaper in rabbits than in cows. The replacement of antibody genes in cows and in rabbits is partly achieved [16]. Human antibodies have been obtained in immunized transgenic cows $[5,17]$ although the cow blood contained a mixture of fully human and chimeric cow-human polyclonal antibodies. The fully human antibodies can be purified from cow blood. The production of the only human antibodies awaits for the complete inactivation of the corresponding cow immunoglobulin genes. The implementation of pigs is perhaps about to be abandoned and the chicken projects is just starting. Moreover, the two complementary species rabbits and cows might be sufficient to meet the demand of the market.

The cows are developed by a US company, Hematech, whereas rabbits originally studied by THP (USA) are now in the hands of Roche (Switzerland). Pigs started being studied by Revivicor (USA) and chicken by Origen (USA).

Antibody sales were of $\$ 10$ billion in 2005, \$26 billion in 2007 and are expected to reach $\$ 50$ billion in 2013 (data from Datamonitor). It is expected than in 5 years or more, the human PABs from cows and rabbits will start having a significant impact on human health and representing an important part of the antibody market.

The implementation of genetically modified organisms (GMO) is a matter of controversy in some countries, especially in the European Union. GM plants are considered being able to disseminate in an uncontrolled manner and threatening human health. These problems appear overestimated due in part to a misinformation of public opinion and this hampers the development of this biotechnology which started being beneficial for a number of people in the world. The implementation of transgenic animals for applications in medicine and alimentation fields is close to become a reality [18]. It is expected that the GM animals will be rejected as the GM plants in the European Union. Most of the GM animals have less chance to disseminate than GM plants but GM animals are raising specific animal welfare problems. The European Union thus decided to support the Pegasus project (http://www.projectpegasus.eu) which aims at studying in depth the various aspects of three projects based on the use of transgenic animals. The project depicted in the present paper is the matter of one the three Pegasus case studies. The production of human PABs in animals has been retained as a relevant case. Indeed, it is expected to be significantly beneficial for human but it also implies relatively strong genetic modifications of animals and the collection of large amount of blood. The results of the Pegasus studies will be presented to Europeans and will be the matter of debates.

\section{ACKNOWLEDGEMENTS}

This work was supported by the Pegasus project

(http://www.projectpegasus.eu) of the European Union.

\section{REFERENCES}

[1] Houdebine, L.M. (2009) Production of pharmaceutical proteins by transgenic animals. Comparative Immunology, Microbiology \& Infectious Diseases, 32, 107-121. doi:10.1016/j.cimid.2007.11.005

[2] Rehbinder, E., et al. (2009) Pharming. Promises and risks of biopharmaceuticals derived from genetically modified plants and animals. Springer-Verlag, Berlin.

[3] Zhu, L., et al. (2005) Production of human monoclonal antibody in eggs of chimeric chickens. Nature Biotechnolology, 23, 1159-1169.

[4] Lonberg, N. (2005) Human antibodies from transgenic animals. Nature Biotechnology, 23, 1117-1125. doi:10.1038/nbt1135

[5] Kuroiwa, Y., et al. (2009) Antigen-specific human polyclonal antibodies from hyperimmunized cattle. $\mathrm{Na}$ ture Biotechnology, 27, 173-181. doi:10.1038/nbt.1521

[6] Geurts, A.M., et al. (2009) Knockout rats via embryo microinjection of zinc-finger nucleases. Science, 325, 433. doi:10.1126/science. 1172447

[7] Boch, J. (2011) TALEs of genome targeting. Nature Biotechnology, 29, 135-136. doi:10.1038/nbt.1767

[8] Kuroiwa, Y., et al. (2004) Sequential targeting of the genes encoding immunoglobulin- $\mu$ and prion protein in cattle. Nature Genetics, 36, 775-780. doi: $10.1038 / \mathrm{ng} 1373$

[9] Richt, J.A., et al. (2007) Production of cattle lacking prion protein. Nature Biotechnology, 25, 132-138. doi: $10.1038 / \mathrm{nbt} 1271$

[10] Newcombe, C. and Newcombe, A.R. (2007) Antibody production: Polyclonal-derived biotherapeutics. Journal of Chromatography B, 848, 2-7. doi:10.1016/j.jchromb.2006.07.004

[11] Mendicino, M., et al. (2011) Generation of antibody- and B cell-deficient pigs by targeted disruption of the J-region gene segment of the heavy chain locus. Transgenic Research, 20, 625-641. doi:10.1007/s11248-010-9444-z

[12] Ramsoondar, J., et al. (2011) Targeted disruption of the porcine immunoglobulin kappa light chain locus. Transgenic Research, 20, 643-653. doi:10.1007/s11248-010-9445-y

[13] Flisikowska, T., et al. (2011) Efficient immunoglobulin gene disruption and targeted replacement in rabbit using zinc finger nucleases. PLOS ONE (in press).

[14] Leighton, P. and Etches, R. (2007) Human polyclonal antibodies from transgenic chickens.

http://www.iptonline.com/articles/public/IPT_24_p48non print.pdf

[15] Keefer, C.L., Pommer, J. and Robl, J.M. (2007) The role 
of transgenic livestock in the treatment of human disease. Council for Agriculture Science and Technology, 35, $1-12$.

[16] Buelow, R. and van Schooten, W. (2007) Ernst schering foundation symposium proceedings, Springer-Verlag, Berlin.
[17] Echelard, Y. (2009) Year of the ox. Nature Biotechnology, 27, 146-147. doi:10.1038/nbt0209-146

[18] Houdebine, L.M. (2010) Meeting report UC Davis transgenic animal research conference VII. Transgenic Research, 19, 127-130. doi:10.1007/s11248-009-9325-5 